\title{
THE STRUCTURE OF THE UNIT GROUP OF SOME GROUP ALGEBRAS
}

\section{ZS. BALOGH}

Received 27 July, 2020

\begin{abstract}
Let $F M$ be the group algebra of the modular 2-group $M$ over a finite field $F$ of characteristic two. In the present note we establish the structure of the unit group of the group algebra $F M$ and verify the question of Johnson.
\end{abstract}

2010 Mathematics Subject Classification: 16S34; $16 \mathrm{U} 60$

Keywords: group ring, group of units, unitary subgroup

\section{INTRODUCTION AND RESULTS}

Let $F$ be a field of characteristic $p$ and let $G$ be a group such that $G$ contains an element of order $p$. Let $U(F G)$ be the group of units of the group algebra $F G$. It is easy to see that $U(F G)=U(F) \times V(F G)$, in which

$$
V(F G):=\left\{x=\sum_{g \in G} \alpha_{g} g \in U(F G) \mid \chi(x)=\sum_{g \in G} \alpha_{g}=1\right\},
$$

where $\chi(x)$ is the augmentation of $x \in F G$ (see [8, Chapters 2-3, p. 194-196]).

The structure of the group of units and its subgroup $V(F G)$ has been investigated by several authors, but the complete description is known only for certain group algebras (for example, see [1,2,10-13,15,16,18-21]). For an overview in this topic we recommend the survey paper [8].

Let $\zeta(G)$ be the center and let $G^{\prime}$ be the commutator subgroup of $G$, respectively. It is well known [9, Theorem 2], if $F G$ is a modular group algebra, then $G \cap \zeta(V(F G))=\zeta(G)$ and $G \cap V(F G)^{\prime}=G^{\prime}$. The question whether $G \cap V(F G)^{p}=$ $G^{p}$ is due to Johnson [15]. The Johnson's question was affirmatively confirmed for nonabelian groups in the following cases: (i) the group of exponent $p$ and order $p^{3}$ [15, Theorem 7]; (ii) $G$ is a finite $p$-group ( $p$ is an odd prime) with Frattini subgroup of order $p$ [4]; and (iii) $G$ is the modular 2-group of order 16 and $F$ is the field of 2 elements [14, Theorem 2]. The structure of elements of order two in $V(F G)$, where

The research was supported by UAEU Research Start-up Grant No. G00002968. 
$G$ is a 2-group of maximal class and $F$ is the field of elements two, was described in [5].

Let

$$
M_{n}=\left\langle a, b \mid a^{2^{n-1}}=b^{2}=1,(a, b)=a^{2^{n-2}}\right\rangle=\langle a\rangle \rtimes\langle b\rangle, \quad(n \geq 4)
$$

be the modular 2-group. The group $M_{n}$ appears very frequently in the investigation of the group of units $[3,6,7,14,17]$.

In the present note the structure of $V\left(F M_{n}\right)$ is established and affirmative answer for the Johnson's question is provided.

Theorem 1. Let $M_{n}$ be the modular group given in (1.1). If $F$ is a field with $|F|=2^{m} \geq 2$, then $V\left(F M_{n}\right)$ is a central extension of $C_{2}^{3 m 2^{n-3}}$ by

$$
C_{2^{n-2}}^{m} \times C_{2}^{7 m 2^{n-4}} \times \prod_{i=0}^{n-5} C_{2^{n-i-3}}^{2^{i} m}
$$

Corollary 1. Let $M_{n}$ be the modular group of order $2^{n}$. If $F$ is a field with $|F|=p^{m} \geq 2$, then

$$
M_{n} \cap V\left(F M_{n}\right)^{2}=M_{n}^{2}
$$

\section{PROOF}

Let $H$ be a normal subgroup of a finite $p$-group $G$. The ideal of $F G$ generated by the set $\{h-1 \mid h \in H\}$ is denoted by $I(H)$. Let $G\left[p^{i}\right]$ denote the subgroup of $G$ generated by the elements of order $p^{i}$. We use the notation $G^{p^{i}}$ for the subgroup $\left\langle g^{p^{i}} \mid g \in G\right\rangle$. Set $x^{g}:=g^{-1} x g$, where $g \in G$ and $x \in F G$. Let $\widehat{S}=\sum_{s \in S} s \in F G$, where $S \subseteq G$ is a finite subset and let $|S|$ denote the cardinality of $S$. Furthermore, the order of $g \in G$ will be denoted by $|g|$.

If $G$ is an abelian $p$-group, then the number of subgroups of order $p^{i}$ in the decomposition of $G$ into a direct product of cyclic groups will be denoted by $f_{i}(G)$.

Lemma 1. Let $F$ be a field with $|F|=p^{m} \geq p$. If $G$ is a finite abelian $p$-group, then

(i) $V(F G)^{p}=V\left(F G^{p}\right)$;

(ii) $V(F G)[p]=1+I(G[p])$; and

(iii) $f_{i}(V(F G))=m\left(\left|G^{p^{i-1}}\right|-2\left|G^{p^{i}}\right|+\left|G^{p^{i+1}}\right|\right)$.

Proof. (i) If $u=\sum_{g \in G} \alpha_{g} g \in V(F G)$, then $u^{p}=\sum_{g \in G} \alpha_{g}^{p} g^{p} \in V\left(F G^{p}\right)$, so $V(F G)^{p} \subseteq V\left(F G^{p}\right)$.

Let $u=\sum_{g \in G^{p}} \alpha_{g} g \in V\left(F G^{p}\right)$. Obviously, the mapping $\tau(\alpha)=\alpha^{p}$ is an automorphism of $F$. Therefore there exists a $\beta_{g} \in F$ and $h \in G$ for every $g \in G^{p}$ such that $\beta_{g}^{p}=\alpha_{g}$ and $h^{p}=g$. We have that $u=\sum_{g \in G^{p}} \alpha_{g} g=\sum_{h \in G} \beta_{g}^{p} h^{p} \in V(F G)^{p}$, which completes the proof.

(ii) If $u \in 1+I(G[p])$, then $u^{p}=1$ and $1+I(G[p]) \subseteq V(F G)[p]$. 
Let $u \in V(F G)[p]$. Clearly, $u-1$ can be written as $x_{1} h_{1}+x_{2} h_{2}+\cdots+x_{s} h_{s}$ for some $s$, where $x_{i} \in F G[p]$ and the set $\left\{h_{i}\right\}$ is a complete set of right coset representatives of $G[p]$ in $G$. We have that $x_{1}^{p} h_{1}^{p}+x_{2}^{p} h_{2}^{p}+\cdots+x_{s}^{p} h_{s}^{p}=(u-1)^{p}=u^{p}-1=0$. Suppose that $h_{i}^{p}=h_{j}^{p}$ for some $i, j$ and $i \neq j$. Clearly, $h_{i} h_{j}^{-1} \in G[p]$ which is impossible. Without loss of generality we can assume that $h_{1}=1$ and $h_{i}^{p} \neq 1$ if $1<i \leq s$. Therefore $x_{i}^{p}=0$ if $1<i \leq s$ and $u-1 \in I(G[p])$ which proves that $V(F G)[p] \subseteq 1+I(G[p])$.

(iii) It is true when $|F|=p[18$, Theorem 2.4]. Now we extend it to any finite field.

If $V(F G)=\left\langle a_{1}\right\rangle \times \cdots \times\left\langle a_{s}\right\rangle$, then $V(F G)[p]=\left\langle a_{1}^{b_{1}-1}\right\rangle \times\left\langle a_{2}^{b_{2}-1}\right\rangle \times \cdots \times$ $\left\langle a_{s}^{b_{s}-1}\right\rangle$ in which $b_{j}=\left|a_{j}\right|$. The number of elements in $1+I(G[p])$ equals $|I(G[p])|$. Evidently, $I(G[p])$ can be considered as a vector space over $F$ with the basis $\{u(h-1) \mid u \in T(G / G[p]), h \in G[p]\}$ in which $T(G / G[p])$ is a complete set of right coset representatives of $G[p]$ in $G$. Thus

$$
|I(G[p])|=p^{m \frac{|G|}{|G| p] \mid}(G[p]-1)}=p^{m\left(|G|-\left|G^{p}\right|\right)} .
$$

According to part (ii), the $p$-rank of $V(F G)$ is $m\left(|G|-\left|G^{p}\right|\right)$.

The part (i) shows that the $p$-rank of $V(F G)^{p}$ is $m\left(\left|G^{p}\right|-\left|G^{p^{2}}\right|\right)$, so

$$
f_{1}(V(F G))=m\left(|G|-\left|G^{p}\right|\right)-m\left(\left|G^{p}\right|-\left|G^{p^{2}}\right|\right)=m\left(|G|-2\left|G^{p}\right|+\left|G^{p^{2}}\right|\right) .
$$

The proof can be easily completed using part (i) and induction on $V\left(F G^{p^{i}}\right)$.

Lemma 2. If $F$ is a field with $|F|=2^{m} \geq 2$, then

$$
\zeta(V)=V\left(F \zeta\left(M_{n}\right)\right) \times N
$$

where $N \cong C_{2}^{3 m 2^{n-3}}$ and $f_{i}\left(V\left(F \zeta\left(M_{n}\right)\right)\right)= \begin{cases}m & \text { if } i=n-2 ; \\ m \cdot 2^{n-3-i} & \text { if } \quad i<n-2 .\end{cases}$

Proof. Let $C_{g}$ be the conjugacy class of $g \in M_{n} \backslash \zeta\left(M_{n}\right)$. Clearly, $\left|C_{g}\right|=2$, $M_{n}^{\prime}=\left\{1, a^{2^{n-2}}\right\}$ and $\widehat{C_{g}}=g \widehat{M_{n}^{\prime}}$. Let $N$ be defined by

$$
N=\left\langle 1+\beta_{i} a^{2 i+1} \widehat{M_{n}^{\prime}} \mid 0 \leq i<2^{n-3}, \beta_{i} \in F\right\rangle \times\left\langle 1+\gamma_{i} a^{i} b \widehat{M_{n}^{\prime}} \mid 0 \leq i<2^{n-2}, \gamma_{i} \in F\right\rangle .
$$

Since $\left(1+x \widehat{M_{n}^{\prime}}\right)\left(1+y \widehat{M_{n}^{\prime}}\right)=1+x \widehat{M_{n}^{\prime}}+y \widehat{M_{n}^{\prime}}$ and $\left(1+x \widehat{M_{n}^{\prime}}\right)^{2}=1$ for every $x, y \in F M_{n}$, the group $N \cong C_{2}^{3 m 2^{n-3}}$ is an elementary abelian 2-group and

$$
\zeta(V)=V\left(F \zeta\left(M_{n}\right)\right) \times N .
$$

Indeed, $V\left(F \zeta\left(M_{n}\right)\right) \times N \subseteq \zeta(V)$. Since $\zeta\left(M_{n}\right)=M_{n}^{2}$, each element $x \in \zeta(V)$ can be written as $x=x_{1}+x_{2}$ in which

$$
x_{1}=\sum_{i=0}^{2^{n-2}-1} \alpha_{i} a^{2 i}, \quad x_{2}=\sum_{i=0}^{2^{n-3}-1} \beta_{i} a^{2 i+1} \widehat{M^{\prime}}+\sum_{i=0}^{2^{n-2}-1} \gamma_{i} a^{i} b \widehat{M}^{\prime}, \quad\left(\alpha_{i}, \beta_{i}, \gamma_{i} \in F\right) \text {. }
$$

It is clear, that the augmentation of $x_{2}$ equals 0 therefore $x_{1}$ is an invertible element with augmentation 1 . Obviously, $1+a^{2 i} \cdot x_{2} \in N$ therefore $x_{1}^{-1} x=x_{1}^{-1}\left(x_{1}+x_{2}\right)=$ 
$1+x_{1}^{-1} x_{2} \in N . \quad$ Since $V\left(F \zeta\left(M_{n}\right)\right) \cap N=\{1\}$ we have proved that $\zeta(V) \subseteq$ $V\left(F \zeta\left(M_{n}\right)\right) \times N$.

Since $\zeta\left(M_{n}\right)=M_{n}^{2} \cong C_{2^{n-2}}$, Lemma 1(iii) ensures that

$$
f_{i}\left(V\left(F \zeta\left(M_{n}\right)\right)\right)=m\left(2^{n-1-i}-2 \cdot 2^{n-2-i}+2^{n-3-i}\right)=m 2^{n-i-3}
$$

for $i<n-2$ and $f_{i}\left(V\left(F \zeta\left(M_{n}\right)\right)\right)=m$ for $i=n-2$.

Lemma 3. Let $F$ be a field with $|F|=2^{m} \geq 2$. Then $|\zeta(V)|=2^{5 m 2^{n-3}-m}$ and

$$
\zeta(V) \cong C_{2^{n-2}}^{m} \times C_{2}^{7 m 2^{n-4}} \times \prod_{i=0}^{n-5} C_{2^{n-i-3}}^{2^{i} m}
$$

Proof. According to the previous lemma, $\zeta(V) \cong V\left(F \zeta\left(M_{n}\right)\right) \times N$. Since $|N|=2^{3 m 2^{n-3}}$ and $\left|V\left(F \zeta\left(M_{n}\right)\right)\right|=|F|^{\left|\zeta\left(M_{n}\right)\right|-1}=2^{m\left(2^{n-2}-1\right)}$, we can easily compute that

$$
|\zeta(V)|=2^{3 m 2^{n-3}+m 2^{n-2}-m}=2^{5 m 2^{n-3}-m} .
$$

Finally, using Lemma 2, it is easy to check that

$$
\zeta(V) \cong C_{2^{n-2}}^{m} \times C_{2^{n-3}}^{m} \times C_{2^{n-4}}^{2 m} \times C_{2^{n-5}}^{2^{2} m} \times C_{2^{n-6}}^{2^{3} m} \times \cdots \times C_{2^{2}}^{m 2^{n-5}} \times C_{2}^{7 m 2^{n-4}} .
$$

Proof of Theorem. Each $x \in F M_{n}$ can be written as $x=x_{1}+x_{2} b$, where $x_{1}, x_{2} \in$ $F\langle a\rangle($ see (1.1)) and

$$
x^{2}=x_{1}^{2}+x_{2} x_{2}^{b}+\left(x_{1}+x_{1}^{b}\right) x_{2} b .
$$

Obviously, we can write $x_{1}=y_{1}+y_{2} a$ and $x_{2}=z_{1}+z_{2} a$, where $y_{1}, y_{2}, z_{1}, z_{2} \in F\left\langle a^{2}\right\rangle$, so

$$
\begin{gathered}
x_{1}+x_{1}^{b}=y_{2} a\left(1+a^{2^{n-2}}\right) \in I\left(M_{n}^{\prime}\right) \quad \text { and } \\
x_{2} x_{2}^{b}=z_{1}^{2}+z_{2}^{2} a^{2^{n-2}+2}+z_{1} z_{2} a\left(1+a^{2^{n-2}}\right) \in \zeta\left(F M_{n}\right) .
\end{gathered}
$$

Consequently, $\left(x_{1}+x_{1}^{b}\right) x_{2} b \in I\left(M_{n}^{\prime}\right) \subseteq \zeta\left(F M_{n}\right)$ and $x^{2} \in \zeta\left(V\left(F M_{n}\right)\right)$ for every $x \in V\left(F M_{n}\right)$. Hence $V\left(F M_{n}\right) / \zeta(V)$ is an elementary abelian 2-group of order $2^{3 m 2^{n-3}}$ and

$$
\zeta(V) \cong C_{2^{n-2}}^{m} \times C_{2^{n-3}}^{m} \times C_{2^{n-4}}^{2 m} \times C_{2^{n-5}}^{2^{2} m} \times C_{2^{n-6}}^{2^{3} m} \times \cdots \times C_{2^{2}}^{m 2^{n-5}} \times C_{2}^{7 m 2^{n-4}}
$$

by Lemma 3 which is the desired conclusion.

Proof of Corollary. Since $V\left(F M_{n}\right)^{2} \subseteq \zeta(V)$ and $\zeta\left(M_{n}\right)=M_{n}^{2}$,

$$
M_{n} \cap V\left(F M_{n}\right)^{2} \subseteq M_{n} \cap \zeta(V) \subseteq \zeta\left(M_{n}\right)=M_{n}^{2} .
$$




\section{ACKNOWLEDGEMENT}

The author would like to gratefully acknowledge the editors and reviewers for their careful reading of the manuscript and their many insightful comments and suggestions.

\section{REFERENCES}

[1] S. F. Ansari and M. Sahai, "The group of units of group algebras of groups $D_{30}$ and $C_{3} \times D_{10}$ over a finite field of characteristic 3," Int. Electron. J. Algebra, 2020.

[2] S. F. Ansari and M. Sahai, "Unit groups of group algebras of groups of order 20," Quaestiones Mathematicae, 2020, doi: 10.2989/16073606.2020.1727583.

[3] Z. Balogh, "On unitary subgroups of group algebras," Int. Electron. J. Algebra, 2020.

[4] Z. Balogh and A. Bovdi, "Group algebras with unit group of class p," Publ. Math. Debrecen, vol. 65, no. 3-4, pp. 261-268, 2004.

[5] Z. Balogh and A. Bovdi, "On units of group algebras of 2-groups of maximal class," Comm. Algebra, vol. 32, no. 8, pp. 3227-3245, 2004, doi: 10.1081/AGB-120039288. [Online]. Available: https://doi.org/10.1081/AGB-120039288

[6] Z. Balogh and V. Bovdi, "The isomorphism problem of unitary subgroups of modular group algebras," Publ. Math. Debrecen, vol. 97, no. 1-2, pp. 27-39, 2020.

[7] A. Bovdi and L. Erdei, "Unitary units in modular group algebras of groups of order 16," Technical Reports, Universitas Debrecen, Dept. of Math., L. Kossuth Univ., vol. 4, no. 157, pp. 1-16, 1996.

[8] A. Bovdi, "The group of units of a group algebra of characteristic p," Publ. Math. Debrecen, vol. 52, no. 1-2, pp. 193-244, 1998.

[9] D. B. Coleman, "On the modular group ring of a p-group," Proc. Amer. Math. Soc., vol. 15, pp. 511-514, 1964, doi: 10.2307/2034735. [Online]. Available: https: //doi-org.uaeu.idm.oclc.org/10.2307/2034735

[10] L. Creedon and J. Gildea, "The structure of the unit group of the group algebra $\mathbb{F}_{2^{k}} D_{8}$, , Canad. Math. Bull., vol. 54, no. 2, pp. 237-243, 2011, doi: 10.4153/CMB-2010-098-5. [Online]. Available: https://doi.org/10.4153/CMB-2010-098-5

[11] J. Gildea, "The structure of the unit group of the group algebra $F_{3^{k}}\left(C_{3} \times D_{6}\right)$," Comm. Algebra, vol. 38, no. 9, pp. 3311-3317, 2010, doi: 10.1080/00927872.2010.482552.

[12] J. Gildea, "Units of group algebras of non-abelian groups of order 16 and exponent 4 over $\mathbb{F}_{2^{k}}$," Results Math., vol. 61, no. 3-4, pp. 245-254, 2012, doi: 10.1007/s00025-011-0094-0. [Online]. Available: https://doi.org/10.1007/s00025-011-0094-0

[13] J. Gildea and R. Taylor, "Units of the group algebra of the group $C_{n} \times D_{6}$ over any finite field of characteristic 3," Int. Electron. J. Algebra, vol. 24, pp. 62-67, 2018, doi: 10.24330/ieja.440205. [Online]. Available: https://doi-org.uaeu.idm.oclc.org/10.24330/ieja.440205

[14] L. R. Ivory, "A note on normal complements in $\bmod p$ envelopes," Proceedings of the American Mathematical Society, vol. 79, no. 4, pp. 9-12, 1980.

[15] D. L. Johnson, "The modular group-ring of a finite p-group," Proc. Amer. Math. Soc., vol. 68, no. 1, pp. 19-22, 1978, doi: 10.2307/2040900. [Online]. Available: https://doi-org.uaeu.idm.oclc.org/10.2307/2040900

[16] N. Makhijani, R. K. Sharma, and J. B. Srivastava, "Units in finite dihedral and quaternion group algebras," J. Egyptian Math. Soc., vol. 24, no. 1, pp. 5-7, 2016, doi: 10.1016/j.joems.2014.08.001. [Online]. Available: https://doi-org.uaeu.idm.oclc.org/10.1016/j.joems.2014.08.001

[17] Z. Raza and M. Ahmad, "On the unitary units of the group algebra $\mathbb{F}_{2^{m}} M_{16}$," J. Algebra Appl., vol. 12, no. 8, pp. 1350 059, 7, 2013, doi: 10.1142/S021949881350059X. [Online]. Available: https://doi.org/10.1142/S021949881350059X 
[18] R. Sandling, "Group rings of circle and unit groups," Math. Z., vol. 140, pp. 195-202, 1974, doi: 10.1007/BF01214162. [Online]. Available: https://doi.org/10.1007/BF01214162

[19] R. K. Sharma, J. B. Srivastava, and M. Khan, "The unit group of $F A_{4}$," Publ. Math. Debrecen, vol. 71, pp. 21-26, 072007.

[20] R. K. Sharma, J. B. Srivastava, and M. Khan, "The unit group of $F_{4}$," Acta Math. Hungar., vol. 118, no. 1-2, pp. 105-113, 2008, doi: 10.1007/s10474-007-6169-4. [Online]. Available: https://doi-org.uaeu.idm.oclc.org/10.1007/s10474-007-6169-4

[21] G. Tang, Y. Wei, and Y. Li, "Unit groups of group algebras of some small groups," Czechoslovak Math. J., vol. 64(139), no. 1, pp. 149-157, 2014, doi: 10.1007/s10587-014-0090-0. [Online]. Available: https://doi-org.uaeu.idm.oclc.org/10.1007/s10587-014-0090-0

\section{Author's address}

\section{Zs. Balogh}

Department of Math. Sci., College of Science, United Arab Emirates University, Al Ain, United Arab Emirates,

E-mail address: baloghzsa@gmail.com 